\title{
Pathogenesis Concept Of Extracranial Dissections In Iran
}

Kavian Ghandehari, MD FLSP RCPC,

Stroke Subspecialist, Director of Comprehensive Stroke Center, M ashhad UMS, Iran

Background: Dissection of Extracranial Internal Carotid Artery (EICA) and Extracranial Vertebral Artery (EVA) is an amportant cause of brain infarction with miscellaneous etiologies around the world.

Methods: A prospective observational clinical study was conducted in Ghaem Hospital, Mashhad, Iran between 2008-2016. Diagnosis of brain infarction and TIA was made by stroke neurologist. Detection of EICA and EVA dissections were made by performing CT angiography and MR angiography or DSA in the suspected patients. Demographic features, clinical manifestations, territorial involvement, pathophysiology and pathogenesis of dissections were assessed in all of the patients. Pathogenesis of dissections was classified as Idiopathic, Trumatic, Postural and Genetic categories.

Results: Twenty eight patients ( 21 males, 7 females) were admitted with extracranial arterial dissection. Mean age of males and females with dissection was $39.81 \pm 4.2$ and $35.71 \pm 6.1$ years respectively. Influence of gender on age of the patients was not significant, $p>0.05$. Among patients with extracranial dissection only $3.6 \%$ had atherosclerosis risk factors and $96.4 \%$ had no other cause for brain infarction. $100 \%$ of extracranial dissections in males occured in carotid territory, while $28.6 \%$ of females had dissection in the EVA. The influence of gender in territory of dissection was significant, $p \varangle 0.05$. Idiopathic dissections and genetic susceptibility was found in $10.7 \%$ and $3.6 \%$ of extracranial dissections respectively. $53.5 \%$ of the patienrs had trumatic pathogenesis for extracranial dissections and $32.1 \%$ developed dissection due to special neck postures. Important details in pathophysiology and pathogenesis of extracranial dissections will be presented in the lecture.

Conclusion: Stroke patients with extracranial dissections have characteristic demographic and territorial involvement. Trumatic pathogenesis is the most frequent cause of dissection in Iran followed by neck postural injry.

Key words: Dissection, Etiology, Pathogenesis

\section{DOI: 10.7575/aiac.abcmed.ca1.7}

